OPEN ACCESS

Edited by:

Ana B. Vivas,

CITY College, International Faculty of the University of Sheffield, Greece

Reviewed by:

Panteleimon Giannakopoulos, Université de Genève, Switzerland

Wei Wang,

Stowers Institute for Medical Research, United States

${ }^{*}$ Correspondence: Xi Chen beating_u5@hotmail.com Xinchun Jin xinchunjin@gmail.com

Received: 08 March 2017 Accepted: 10 May 2017 Published: 24 May 2017

Citation:

Liu W-C, Wang X, Zhang X, Chen X and $\operatorname{Jin} X$ (2017) Melatonin Supplementation, a Strategy to Prevent Neurological Diseases Through Maintaining Integrity of Blood Brain Barrier in Old People. Front. Aging Neurosci. 9:165. doi: 10.3389/fnagi.2017.00165

\section{Melatonin Supplementation, a Strategy to Prevent Neurological Diseases through Maintaining Integrity of Blood Brain Barrier in Old People}

\author{
Wen-Cao Liu ${ }^{1}$, Xiaona Wang ${ }^{2,3}$, Xinyu Zhang ${ }^{2,3}$, Xi Chen $^{4 *}$ and Xinchun Jin ${ }^{2,3 *}$ \\ 'Department of Emergency, Shanxi Provincial People's Hospital, Taiyuan, China, 2Jiangsu Key Laboratory of Translational \\ Research and Therapy for Neuro-Psycho-Diseases and Institute of Neuroscience, Department of Neurology, the Second \\ Affiliated Hospital of Soochow University, Suzhou, China, ${ }^{3}$ School of Pharmacy, Key Laboratory of Molecular Pharmacology \\ and Drug Evaluation (Yantai University), Ministry of Education, Yantai University, Yantai, China, ${ }^{4}$ Department of Core Facility, \\ the People's Hospital of Baoan Shenzhen, Shenzhen, China
}

Blood brain barrier (BBB) plays a crucial role in maintaining homeostasis of microenvironment that is essential to neural function of the central nervous system (CNS). When facing various extrinsic or intrinsic stimuli, BBB is damaged which is an early event in pathogenesis of a variety of neurological diseases in old patients including acute and chronic cerebral ischemia, Alzheimer's disease and etc. Treatments that could maintain the integrity of BBB may prevent neurological diseases following various stimuli. Old people often face a common stress of sepsis, during which lipopolysaccharide (LPS) is released into circulation and the integrity of BBB is damaged. Of note, there is a significant decrease of melatonin level in old people and animal. Melatonin has been shown to preserves BBB integrity and permeability via a variety of pathways: inhibition of matrix metalloproteinase-9 (MMP-9), inhibition of NADPH oxidase-2, and impact on silent information regulator 1 (SIRT1) and nucleotide-binding oligomerization domainlike receptor family pyrin domain-containing 3 (NLRP3) inflammasome. More important, a recent study showed that melatonin supplementation alleviates LPS-induced BBB damage in old mice through activating AMP-activated protein kinase (AMPK) and inhibiting gp91phox, suggesting that melatonin supplementation may help prevent neurological diseases through maintaining the integrity of BBB in old people.

Keywords: melatonin, blood brain barrier, neurological diseases, old people, lipopolysaccharide

\footnotetext{
Abbreviations: AMPK, AMP-activated protein kinase; BBB, blood brain barrier; CNS, central nervous system; iNOS, inducible nitric oxide synthase; LPS, lipopolysaccharide; MMP-9, matrix metalloproteinase-9; nNOS, neuronal nitric oxide synthase; NADPH, nicotinamide adenine dinucleotide phosphate; NF- $\kappa \mathrm{B}$, nuclear factor kappa-light-chain-enhancer of activated B cells; NLRP3, nucleotide-binding oligomerization domain-like receptor family pyrin domain-containing 3; ROS, reactive oxygen species; SIRT1, silent information regulator 1; TBI, traumatic brain injury; TJPs, tight junction proteins; TLR4, toll like receptor 4 .
} 


\section{INTRODUCTION}

\section{The Blood Brain Barrier Damage and Neurological Diseases}

The blood brain barrier (BBB) is a regulated interface between the peripheral circulation and the central nervous system (CNS; Jin et al., 2014). BBB, which is composed of cerebral microvascular endothelial cells, neurons, astrocytes, pericytes and the extracellular matrix, plays a key role in maintaining homeostasis of microenvironment that is essential to neural function of the CNS (Hawkins and Davis, 2005). When facing various extrinsic or intrinsic stimuli (Weiss et al., 2009), $\mathrm{BBB}$ is damaged and BBB dysfunction is an early event in pathogenesis of a variety of neurological diseases in old patients including vascular cognitive impairment, amyotrophic lateral sclerosis, Alzheimer's disease, neuropathic pain, brain trauma, acute and chronic cerebral ischemia, multiple sclerosis, and brain infections (Rosenberg, 2012; please see Figure 1). Treatments that could maintain the integrity of $\mathrm{BBB}$ will have important roles in preventing stimuli-produced neurological diseases.

The tight junction proteins (TJPs), composed of occludin, claudin and zo-1, are key components of the BBB (Hawkins and Davis, 2005). It seals the interendothelial cleft forming a continuous blood vessel, leads to high endothelial electrical resistance, and allows low paracellular permeability of watersoluble substances from the blood into brain parenchyma (Stamatovic et al., 2008). Free radicals of oxygen and nitrogen and the proteases, matrix metalloproteinases (MMPs) and cyclooxgyenases, play key roles in the early and delayed BBB disruption as the neuroinflammatory response progresses (Liu and Rosenberg, 2005). During an injury, free radicals and proteases attacked the cell membranes and degraded the TJPs between endothelial cells and the integrity of BBB is damaged

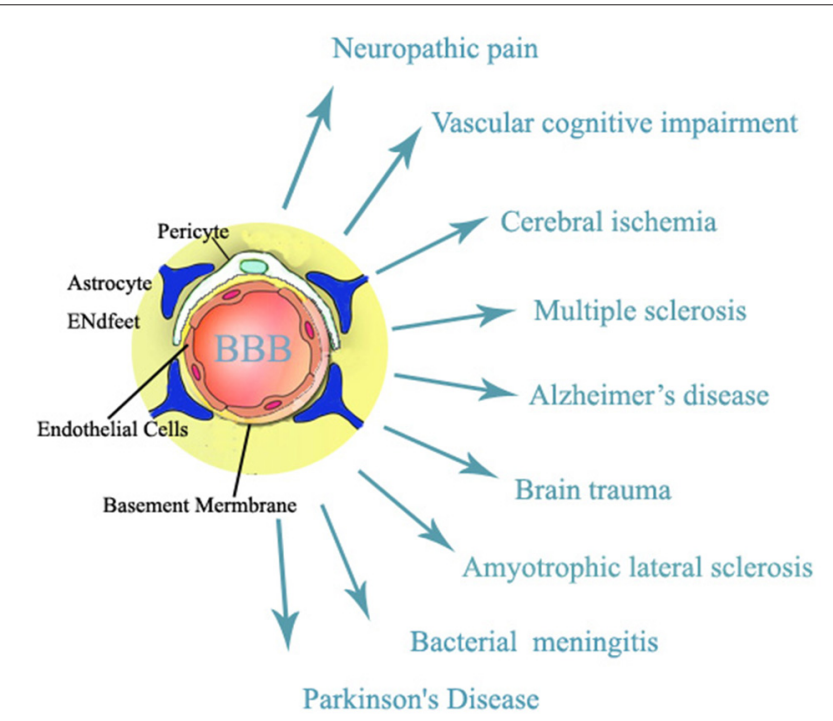

FIGURE 1 | Structure of blood brain barrier (BBB) which is related to neurological diseases.
(Jin et al., 2013; Liu et al., 2016; Wang et al., 2016). It is worth of note, death of endothelial cells of microvessels is also a major contributor to the disruption of BBB integrity (Simard et al., 2007). Therefore, protective effect on intergrity of BBB should consider both death of endothelial cells of microvessels and degradation of TJPS.

\section{BBB Disruption Induced by Lipopolysaccharide (LPS)}

Lipopolysaccharide (LPS) could produce neuroinflammation (Shi, 2015), promoting the generation of reactive oxygen species (ROS) in cerebral microvascular endothelial cells and BBB disruption (Seok et al., 2013). Worth of note, LPS has been shown to increase BBB permeability in vitro (Nonaka et al., 2004) and compromise BBB integrity in young (Ruiz-Valdepeñas et al., 2011; Zhou T. et al., 2014) and old mice (Wang et al., 2017). More interesting, LPS has been shown to induce BBB dysfunction via nicotinamide adenine dinucleotide phosphate (NADPH) oxidase-derived ROS (Liu et al., 2012; Zhao et al., 2014). NADPH oxidases, a major source of ROS generation in the brain, critically contributes to BBB disruption under various neurological disorders (Kahles et al., 2007). Of note, gp91 ${ }^{\text {phox }}$ is the catalytic subunit of NADPH oxidase and BBB disruption is significantly reduced in gp91 ${ }^{\text {phox }}$ knockout mice compared to wild-type mice after stroke (Kahles et al., 2007) and reduction of gp91 9 phox expression has shown protective effect against ischemia-induced brain injury and BBB damage (Liu et al., 2008, 2011). More importantly, Wang et al. (2017) showed that LPS increased gp91 ${ }^{\text {phox }}$ expression in both endothelial cells and in old mice, suggesting that gp91 ${ }^{\text {phox }}$ up-regulation may be an important mechanism responsible for LPS-induced BBB permeability increase in old mice.

\section{Relationship between Melatonin and Aging}

Melatonin, which is produced mainly in the pineal gland, retina and the gastrointestinal tract, plays important roles in many physiological and biochemical functions (Bubenik and Konturek, 2011), such as acting as an anti-inflammatory and immunoregulating molecule as well as a circadian rhythm regulator (Manchester et al., 2015). Melatonin is a potent free radical scavenger, lack of melatonin may result in decreased antioxidant function in the old people which have significant influence not only on aging per se but also on the incidence or severity of age-related diseases (Karasek, 2004). In addition, oxygen radical detoxification processes was significantly decreased during aging and there was a obvious downregualtion in pineal biosynthetic activity in aging hamster (Bubenik and Konturek, 2011). More interesting, melatonin levels in serum and brain decline as a result of aging (Bubenik and Konturek, 2011; Hill et al., 2013). In addition, melatonin has been reported to regulate aging and neurodegeneration through energy metabolism, epigenetics, autophagy and circadian rhythm pathways (Jenwitheesuk et al., 2014).

\section{Beneficial Role of Melatonin in Sepsis}

Sepsis is a systemic inflammatory response to infection that causes severe neurological complications (Zhao et al., 2015) and 
it is a common stress that old people often face (Martin et al., 2006), in which LPS is released into circulation (Shukla et al., 2014).

Melatonin has been shown to restore the mitochondrial production of ATP in septic mice (López et al., 2006a), block the septic response by disrupting connection of the nuclear factor kappa-light-chain-enhancer of activated B cells (NF- $\mathrm{B}$ ) with nucleotide-binding oligomerization domain-like receptor family pyrin domain-containing 3 (NLRP3) in mice (El Frargy et al., 2015) and improve survival in a zymosan A-induced rat model of sepsis/shock (Reynolds et al., 2003). In addition, melatonin has been shown to protect organs against sepisinuduced injury. For example, melatonin improved cardiac mitochondria and survival rate in rat septic heart injury (Zhang et al., 2013) through inhibition of inducible nitric oxide synthase (iNOS) and preservation of neuronal nitric oxide synthase (nNOS; Ortiz et al., 2014) and attenuated sepsis-induced cardiac dysfunction via a PI3K/Akt-dependent mechanism (An et al., 2016). Furthermore, melatonin protected liver bioenergetics from sepsis-induced damage (Basile et al., 2004), modified cellular stress in the liver of septic mice by reducing ROS and increasing the unfolded protein response (Kleber et al., 2014), protected against sepsis-induced functional and biochemical changes in rat ileum and urinary bladder (Paskaloglu et al., 2004), improved colonic anastomotic healing in a rat experimental sepsis model (Ersoy et al., 2016) and counteracted inducible mitochondrial nitric oxide synthase-dependent mitochondrial dysfunction in skeletal muscle (Escames et al., 2006) and diaphragm (López et al., 2006b) in septic mice.

\section{Melatonin's Effect on LPS-Induced Injury}

Melatonin has been shown to ameliorate LPS-induced brain injury in neonatal rats (Wong et al., 2014), alleviate LPS-induced placental cellular stress response in mice (Wang et al., 2011) as well as LPS-induced hepatic SREBP-1c activation and lipid accumulation in mice (Chen et al., 2011). Of note, melatonin shown protective effect against BBB damage induced by various stimuli, including transient focal cerebral ischemia in mice (Chen et al., 2006), excitotoxic injury in neonatal rats (Moretti et al., 2015) and methamphetamine-induced inflammation (Jumnongprakhon et al., 2016). Therefore, decreased levels of melatonin in old mice may contribute to the BBB disruption when facing various extrinsic or intrinsic stimuli because melatonin has demonstrated its protective effects against LPS-induced injury to the heart (Lu et al., 2015), brain (Carloni et al., 2016), lung (Lee et al., 2009) and liver (Wang et al., 2007) by scavenging a variety of free radicals (Manchester et al., 2015). Interestingly, chronic melatonin treatment has also shown reduction of age-dependent inflammatory process in senescenceaccelerated mice (Rodríguez et al., 2007). In a recent study, Wang et al. (2017) showed that 1 week melatonin treatment significantly alleviated LPS-induced BBB damage accompanied by reduction of occludin and claudin- 5 degradation, suggesting that melatonin supplementation is important in decreasing sepsis and neuroinflammation-induced TJPs degradation as well as $\mathrm{BBB}$ damage.

\section{Possible Molecular Mechanism Underlying Melatonin's Effect on LPS-Induced BBB Damage in Old Mice}

Melatonin has shown protective effect on BBB integrity via a variety of pathways: inhibition of the toll like receptor 4 (TLR4)/NF-кB signaling pathway in neonatal rats (Hu et al., 2017), inhibition of NADPH oxidase-2 (Jumnongprakhon et al., 2016), inhibition of MMP-9 (Alluri et al., 2016), inhibiton of AMP-activated protein kinase (AMPK) activation (Wang et al., 2017) and impact on silent information regulator 1 (SIRT1; Zhao et al., 2015) and NLRP3 inflammasome (Rahim et al., 2017).

\section{AMPK Activation}

AMPK activation has been shown to play important role in maintaining the integrity of BBB (Liu et al., 2012) and it is also reported that LPS inhibits the activation of AMPK, a serine/threonine protein kinase regulating cellular and organismal metabolism (Wang et al., 2017). Interestingly, AMPK activation has been shown to alleviate LPS-induced $\mathrm{BBB}$ disruption in both in vitro cell model (Zhao et al., 2014) and in vivo mice model (Zhou X. et al., 2014; Wang et al., 2017). Activation of AMPK also demonstrated protective effect against diabetes-induced $\mathrm{BBB}$ damage by inhibiting NADPH oxidase expression upregulation in brain capillary endothelial cells (Liu et al., 2012). In a recent study, Wang et al. (2017) showed that AMPK activation by melatonin reduced LPS-induced BBB damage in old mice and AMPK activation by metformin decreased LPS-induced gp $91^{\text {phox }}$ up-regulation in brain capillary endothelial cells (Figure 2). AMPK activation might be important in maintaining the integrity of $\mathrm{BBB}$ in old patients and AMPK dysfunction might play a key role in the initiation and progression of neurological disorders in old people. Therefore, activation of AMPK may be a strategy to reduce neurological disorders following sepsis and neuroinflamation-induced BBB damage in old people.

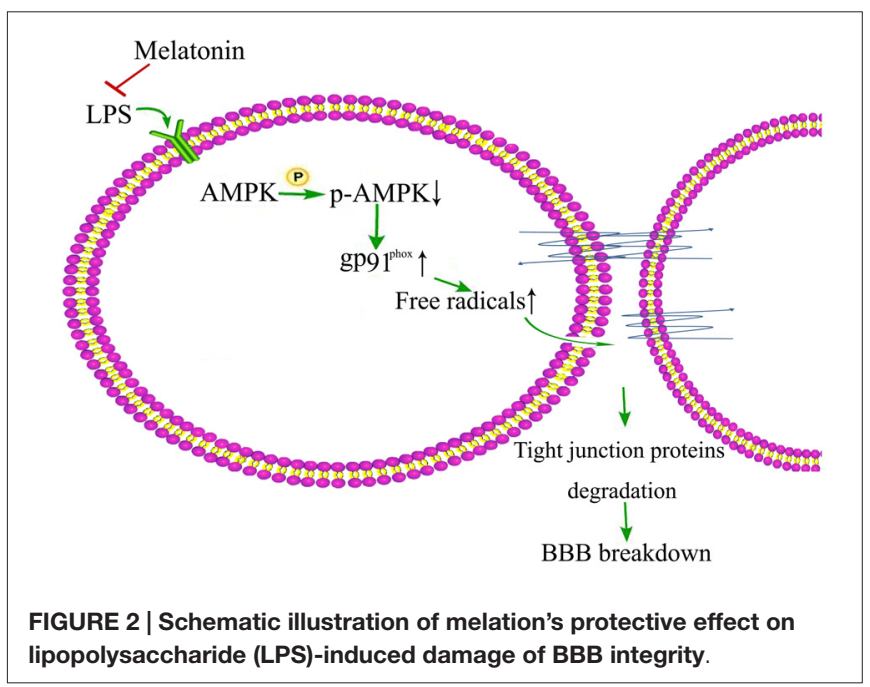




\section{Matrix Metalloproteinase-9 (MMP-9)}

MMP-9 has been shown to play important role in BBB damage (Jin et al., 2013, 2015; Cai et al., 2015) and melatonin has been shown to bind to MMP-9 to act as its endogenous inhibitor. Melatonin treatment provided protection against traumatic brain injury (TBI)-induced BBB hyperpermeability via MMP-9 inhibition (Alluri et al., 2016), indicating its potential as a therapeutic agent for BBB damage.

\section{Silent Information Regulator 1 (SIRT1)}

SIRT1 was reported to be beneficial in sepsis. Using EX527, a SIRT1 inhibitor, the authors figured out that melatonin alleviated $\mathrm{BBB}$ damage in mice which subjected to cecal ligation and puncture via SIRT1 to inhibit inflammation, apoptosis and oxidative stress (Zhao et al., 2015).

\section{NLRP3 Inflammasome}

Aging and sepsis triggered NLRP3 inflammasome activation (Volt et al., 2016), which has been shown to be involved in the innate immune response during inflammation (Rahim et al., 2017). Furthermore, NLRP3 inflammasome activation was showed to be associated with the upregulation of apoptotic signaling pathway in various inflammatory diseases (Volt et al., 2016) and melatonin attenuated subarachnoid hemorrhage-induced BBB damage via attenuating the expressions of NLRP3 (Dong et al., 2016).

\section{Dark Side/Downsides of Melatonin Supplementation}

Although acute toxicity of melatonin is extremely low in both animal and human studies, melatonin may still cause minor adverse effects, such as headache, insomnia and nightmares (Malhotra et al., 2004). Based on previous studies, melatonin could be used as a daily supplement to delay or prevent changes associated with age. However, long-term side effect of melatonin has to be tested, because melatonin has been used as a contraceptive for women which could have reproduction alterations by consumption of melatonin (Voordouw et al., 1992). In addition, there was a decrease in sperm motility in male rats (Gwayi and Bernard, 2002), and long-term administration

\section{REFERENCES}

Alluri, H., Wilson, R. L., Anasooya Shaji, C., Wiggins-Dohlvik, K., Patel, S., Liu, Y., et al. (2016). Melatonin preserves blood-brain barrier integrity and permeability via matrix metalloproteinase-9 inhibition. PLoS One 11:e0154427. doi: 10.1371/journal.pone.0154427

An, R., Zhao, L., Xi, C., Li, H., Shen, G., Liu, H., et al. (2016). Melatonin attenuates sepsis-induced cardiac dysfunction via a PI3K/Akt-dependent mechanism. Basic Res. Cardiol. 111:8. doi: 10.1007/s00395-015-0526-1

Bartsch, H., and Bartsch, C. (1981). Effect of melatonin on experimental tumors under different photoperiods and times of administration. J. Neural Transm. 52, 269-279. doi: 10.1007/bf01256752

Basile, M., Romeo, C., Gitto, E., Spitz, L., Pierro, A., and Eaton, S. (2004). Melatonin protects from, but does not reverse, the effects of mediators of sepsis on liver bioenergetics. Pediatr. Surg. Int. 20, 263-266. doi: 10.1007/s00383-0031123-1 of melatonin inhibited testicular aromatase levels (Luboshitzky et al., 2002). It does not matter to provide old people with daily melatonin to prevent neurological diseases even if these two side effects may happen as they would not have reproduction anymore. Other side effects should be considered, for example, melatonin may accelerate the development of autoimmune conditions (Mattsson et al., 1994), increase atherosclerosis in the aorta in hypercholesterolemic rats (Tailleux et al., 2002) and produce opposite effects in cancer treatment with poorly timed administration (Bartsch and Bartsch, 1981).

\section{Conclusion}

In conclusion, decreased melatonin levels may account for the $\mathrm{BBB}$ damage in old people who often face the common stress of sepsis and neuroinflammation. Melation supplementation treatment significantly inhibits such events. Therefore, continuous daily melatonin supplementation may help prevent sepsis and neuroinflammation-related neurological diseases through maintaining the integrity of $\mathrm{BBB}$ in old people. Since melatonin has low toxicity profile and high efficacy in many pathophysiological states, it should be more commonly tested/used in the medical and veterinary arenas. Further studies are needed to verify the important significance of daily melatonin supplementation in old people.

\section{AUTHOR CONTRIBUTIONS}

$\mathrm{W}$-CL, XW, XZ, XC and XJ wrote the manuscript and $\mathrm{XC}, \mathrm{XJ}$ obtained the funding. XW drew the figures. All authors have approved the final version of this review article.

\section{ACKNOWLEDGMENTS}

This work was supported by National Natural Science Foundation of China (81671145), by Natural Science Foundation of Jiangsu Province of China (L221506415) and by grants from Shenzhen Science and Technology Innovation Commission (JCYJ20150402152005623). This work was also partly supported by Priority Academic Program Development of Jiangsu Higher Education Institutions of China.

Bubenik, G. A., and Konturek, S. J. (2011). Melatonin and aging: prospects for human treatment. J. Physiol. Pharmacol. 62, 13-19.

Cai, H., Mu, Z., Jiang, Z., Wang, Y., Yang, G. Y., and Zhang, Z. (2015). Hypoxiacontrolled matrix metalloproteinase- 9 hyperexpression promotes behavioral recovery after ischemia. Neurosci. Bull. 31, 550-560. doi: 10.1007/s12264-0151533-1

Carloni, S., Favrais, G., Saliba, E., Albertini, M. C., Chalon, S., Longini, M., et al. (2016). Melatonin modulates neonatal brain inflammation through endoplasmic reticulum stress, autophagy, and miR-34a/silent information regulator 1 pathway. J. Pineal Res. 61, 370-380. doi: 10.1111/jpi. 12354

Chen, T. Y., Lee, M. Y., Chen, H. Y., Kuo, Y. L., Lin, S. C., Wu, T. S., et al. (2006). Melatonin attenuates the postischemic increase in blood-brain barrier permeability and decreases hemorrhagic transformation of tissue-plasminogen activator therapy following ischemic stroke in mice. J. Pineal Res. 40, 242-250. doi: 10.1111/j.1600-079x.2005.00307.x 
Chen, X., Zhang, C., Zhao, M., Shi, C.-E., Zhu, R.-M., Wang, H., et al. (2011). Melatonin alleviates lipopolysaccharide-induced hepatic SREBP-1c activation and lipid accumulation in mice. J. Pineal Res. 51, 416-425. doi: 10.1111/j.1600079x.2011.00905.x

Dong, Y., Fan, C., Hu, W., Jiang, S., Ma, Z., Yan, X., et al. (2016). Melatonin attenuated early brain injury induced by subarachnoid hemorrhage via regulating NLRP3 inflammasome and apoptosis signaling. J. Pineal Res. 60, 253-262. doi: 10.1111/jpi.12300

El Frargy, M., El-Sharkawy, H. M., and Attia, G. F. (2015). Use of melatonin as an adjuvant therapy in neonatal sepsis. J. Neonatal Perinatal Med. 8, 227-232. doi: 10.3233/NPM-15814072

Ersoy, O. F., Özkan, N., Özsoy, Z., Kayaoglu, H. A., Yenidogan, E., Çelik, A., et al. (2016). Effects of melatonin on cytokine release and healing of colonic anastomoses in an experimental sepsis model. Ulus. Travma Acil Cerrahi Derg. 22, 315-321. doi: 10.5505/tites.2015.49465

Escames, G., López, L. C., Tapias, V., Utrilla, P., Reiter, R. J., Hitos, A. B., et al. (2006). Melatonin counteracts inducible mitochondrial nitric oxide synthase-dependent mitochondrial dysfunction in skeletal muscle of septic mice. J. Pineal Res. 40, 71-78. doi: 10.1111/j.1600-079x.2005.00281.x

Gwayi, N., and Bernard, R. T. (2002). The effects of melatonin on sperm motility in vitro in Wistar rats. Andrologia 34, 391-396. doi: 10.1046/j.1439-0272.2002. 00522.x

Hawkins, B. T., and Davis, T. P. (2005). The blood-brain barrier/neurovascular unit in health and disease. Pharmacol. Rev. 57, 173-185. doi: 10.1124/pr.57.2.4

Hill, S. M., Cheng, C., Yuan, L., Mao, L., Jockers, R., Dauchy, B., et al. (2013). Agerelated decline in melatonin and its MT1 receptor are associated with decreased sensitivity to melatonin and enhanced mammary tumor growth. Curr. Aging Sci. 6, 125-133. doi: 10.2174/1874609811306010016

Hu, Y., Wang, Z., Pan, S., Zhang, H., Fang, M., Jiang, H., et al. (2017). Melatonin protects against blood-brain barrier damage by inhibiting the TLR4/NF-кB signaling pathway after LPS treatment in neonatal rats. Oncotarget 8 , 31638-31654. doi: 10.18632/oncotarget.15780

Jenwitheesuk, A., Nopparat, C., Mukda, S., Wongchitrat, P., and Govitrapong, P. (2014). Melatonin regulates aging and neurodegeneration through energy metabolism, epigenetics, autophagy and circadian rhythm pathways. Int. J. Mol. Sci. 15, 16848-16884. doi: 10.3390/ijms150916848

Jin, X., Liu, J., and Liu, W. (2014). Early ischemic blood brain barrier damage: a potential indicator for hemorrhagic transformation following tissue plasminogen activator (tPA) thrombolysis? Curr. Neurovasc. Res. 11, 254-262. doi: 10.2174/1567202611666140530145643

Jin, X., Liu, J., Liu, K. J., Rosenberg, G. A., Yang, Y., and Liu, W. (2013). Normobaric hyperoxia combined with minocycline provides greater neuroprotection than either alone in transient focal cerebral ischemia. Exp. Neurol. 240, 9-16. doi: 10.1016/j.expneurol.2012.11.018

Jin, X., Sun, Y., Xu, J., and Liu, W. (2015). Caveolin-1 mediates tissue plasminogen activator-induced MMP-9 up-regulation in cultured brain microvascular endothelial cells. J. Neurochem. 132, 724-730. doi: 10.1111/jnc.13065

Jumnongprakhon, P., Govitrapong, P., Tocharus, C., and Tocharus, J. (2016). Melatonin promotes blood-brain barrier integrity in methamphetamineinduced inflammation in primary rat brain microvascular endothelial cells. Brain Res. 1646, 182-192. doi: 10.1016/j.brainres.2016.05.049

Kahles, T., Luedike, P., Endres, M., Galla, H. J., Steinmetz, H., Busse, R., et al. (2007). NADPH oxidase plays a central role in blood-brain barrier damage in experimental stroke. Stroke 38, 3000-3006. doi: 10.1161/STROKEAHA.107. 489765

Karasek, M. (2004). Melatonin, human aging, and age-related diseases. Exp. Gerontol. 39, 1723-1729. doi: 10.1016/j.exger.2004.04.012

Kleber, A., Kubulus, D., Rössler, D., Wolf, B., Volk, T., Speer, T., et al. (2014). Melatonin modifies cellular stress in the liver of septic mice by reducing reactive oxygen species and increasing the unfolded protein response. Exp. Mol. Pathol. 97, 565-571. doi: 10.1016/j.yexmp.2014.10.009

Lee, Y. D., Kim, J. Y., Lee, K. H., Kwak, Y. J., Lee, S. K., Kim, O. S., et al. (2009). Melatonin attenuates lipopolysaccharide-induced acute lung inflammation in sleep-deprived mice. J. Pineal Res. 46, 53-57. doi: 10.1111/J.1600-079X.2008. 00621.x

Liu, W., Chen, Q., Liu, J., and Liu, K. J. (2011). Normobaric hyperoxia protects the blood brain barrier through inhibiting Nox2 containing NADPH oxidase in ischemic stroke. Med. Gas Res. 1:22. doi: 10.1186/2045-9912-1-22
Liu, K. J., and Rosenberg, G. A. (2005). Matrix metalloproteinases and free radicals in cerebral ischemia. Free Radic. Biol. Med. 39, 71-80. doi: 10.1016/j. freeradbiomed.2005.03.033

Liu, W., Sood, R., Chen, Q., Sakoglu, U., Hendren, J., Cetin, O., et al. (2008). Normobaric hyperoxia inhibits NADPH oxidase-mediated matrix metalloproteinase- 9 induction in cerebral microvessels in experimental stroke. J. Neurochem. 107, 1196-1205. doi: 10.1111/j.1471-4159.2008. 05664.x

Liu, J., Weaver, J., Jin, X., Zhang, Y., Xu, J., Liu, K. J., et al. (2016). Nitric oxide interacts with caveolin-1 to facilitate autophagy-lysosome-mediated claudin-5 degradation in oxygen-glucose deprivation-treated endothelial cells. Mol. Neurobiol. 53, 5935-5947. doi: 10.1007/s12035-015-9504-8

Liu, C., Wu, J., and Zou, M. H. (2012). Activation of AMP-activated protein kinase alleviates high-glucose-induced dysfunction of brain microvascular endothelial cell tight-junction dynamics. Free Radic. Biol. Med. 53, 1213-1221. doi: 10.1016/j.freeradbiomed.2012.07.003

López, L. C., Escames, G., Ortiz, F., Ros, E., and Acuña-Castroviejo, D. (2006a). Melatonin restores the mitochondrial production of ATP in septic mice. Neuro. Endocrinol. Lett. 27, 623-630.

López, L. C., Escames, G., Tapias, V., Utrilla, P., Leon, J., and AcuñaCastroviejo, D. (2006b). Identification of an inducible nitric oxide synthase in diaphragm mitochondria from septic mice: its relation with mitochondrial dysfunction and prevention by melatonin. Int. J. Biochem. Cell Biol. 38, 267-278. doi: 10.1016/j.biocel.2005.09.008

Lu, Q., Yi, X., Cheng, X., Sun, X., and Yang, X. (2015). Melatonin protects against myocardial hypertrophy induced by lipopolysaccharide. In Vitro Cell. Dev. Biol. Anim. 51, 353-360. doi: 10.1007/s11626-014-9844-0

Luboshitzky, R., Shen-Orr, Z., Nave, R., Lavi, S., and Lavie, P. (2002). Melatonin administration alters semen quality in healthy men. J. Androl. 23, 572-578. doi: 10.1002/j.1939-4640.2002.tb02281.x

Malhotra, S., Sawhney, G., and Pandhi, P. (2004). The therapeutic potential of melatonin: a review of the science. MedGenMed 6:46.

Manchester, L. C., Coto-Montes, A., Boga, J. A., Andersen, L. P., Zhou, Z., Galano, A., et al. (2015). Melatonin: an ancient molecule that makes oxygen metabolically tolerable. J. Pineal Res. 59, 403-419. doi: 10.1111/jpi.12267

Martin, G. S., Mannino, D. M., and Moss, M. (2006). The effect of age on the development and outcome of adult sepsis. Crit. Care Med. 34, 15-21. doi: 10.1097/01.ccm.0000194535.82812.ba

Mattsson, R., Hannsson, I., and Holmdahl, R. (1994). Pineal gland in autoimmunity: melatonin-dependent exaggeration of collagen-induced arthritis in mice. Autoimmunity 17, 83-86. doi: 10.3109/08916939409 014661

Moretti, R., Zanin, A., Pansiot, J., Spiri, D., Manganozzi, L., Kratzer, I., et al. (2015). Melatonin reduces excitotoxic blood-brain barrier breakdown in neonatal rats. Neuroscience 311, 382-397. doi: 10.1016/j.neuroscience.2015. 10.044

Nonaka, N., Hileman, S. M., Shioda, S., Vo, T. Q., and Banks, W. A. (2004). Effects of lipopolysaccharide on leptin transport across the blood-brain barrier. Brain Res. 1016, 58-65. doi: 10.1016/j.brainres.2004.04.066

Ortiz, F., García, J. A., Acuña-Castroviejo, D., Doerrier, C., López, A., Venegas, C., et al. (2014). The beneficial effects of melatonin against heart mitochondrial impairment during sepsis: inhibition of iNOS and preservation of nNOS. J. Pineal Res. 56, 71-81. doi: 10.1111/jpi.12099

Paskaloğlu, K., Sener, G., Kapucu, C., and Ayanoğlu-Dülger, G. (2004). Melatonin treatment protects against sepsis-induced functional and biochemical changes in rat ileum and urinary bladder. Life Sci. 74, 1093-1104. doi: 10.1016/j.lfs.2003. 07.038

Rahim, I., Djerdjouri, B., Sayed, R. K., Fernández-Ortiz, M., Fernández-Gil, B., Hidalgo-Gutiérrez, A., et al. (2017). Melatonin administration to wild-type mice and non-treated NLRP3 mutant mice share similar inhibition of the inflammatory response during sepsis. J. Pineal Res. doi: 10.1111/jpi.12410 [Epub ahead of print].

Reynolds, F. D., Dauchy, R., Blask, D., Dietz, P. A., Lynch, D., and Zuckerman, R. (2003). The pineal gland hormone melatonin improves survival in a rat model of sepsis/shock induced by zymosan A. Surgery 134, 474-479. doi: 10.1067/s0039-6060(03)00253-8

Rodríguez, M. I., Escames, G., Löpez, L. C., Löpez, A., García, J. A., Ortiz, F., et al. (2007). Chronic melatonin treatment reduces the age-dependent 
inflammatory process in senescence-accelerated mice. J. Pineal Res. 42, 272-279. doi: 10.1111/j.1600-079x.2006.00416.x

Rosenberg, G. A. (2012). Neurological diseases in relation to the blood-brain barrier. J. Cereb. Blood Flow Metab. 32, 1139-1151. doi: 10.1038/jcbfm. 2011.197

Ruiz-Valdepeñas, L., Martínez-Orgado, J. A., Benito, C., Millán, A., Tolón, R. M., and Romero, J. (2011). Cannabidiol reduces lipopolysaccharide-induced vascular changes and inflammation in the mouse brain: an intravital microscopy study. J. Neuroinflammation 8:5. doi: 10.1186/1742-2094-8-5

Seok, S. M., Kim, J. M., Park, T. Y., Baik, E. J., and Lee, S. H. (2013). Fructose-1,6bisphosphate ameliorates lipopolysaccharide-induced dysfunction of bloodbrain barrier. Arch. Pharm. Res. 36, 1149-1159. doi: 10.1007/s12272-013 $-0129-\mathrm{z}$

Shi, F. D. (2015). Neuroinflammation. Neurosci. Bull. 31, 714-716. doi: 10.1007/s12264-015-1568-y

Shukla, P., Rao, G. M., Pandey, G., Sharma, S., Mittapelly, N., Shegokar, R., et al. (2014). Therapeutic interventions in sepsis: current and anticipated pharmacological agents. Br. J. Pharmacol. 171, 5011-5031. doi: 10.1111/bph. 12829

Simard, J. M., Kent, T. A., Chen, M., Tarasov, K. V., and Gerzanich, V. (2007). Brain oedema in focal ischemeia: molecular pathophysiology and theoretical implications. Lancet Neurol. 6, 258-268. doi: 10.1016/s1474-4422(07)70055-8

Stamatovic, S. M., Keep, R. F., and Andjelkovic, A. V. (2008). Brain endothelial cell-cell junctions: how to "open" the blood brain barrier. Curr. Neuropharmacol. 6, 179-192. doi: 10.2174/157015908785777210

Tailleux, A., Torpier, G., Bonnefont-Rousselot, D., Lestavel, S., Lemdani, M., Caudeville, B., et al. (2002). Daily melatonin supplementation in mice increases atherosclerosis in proximal aorta. Biochem. Biophys. Res. Commun. 293, 1114-1123. doi: 10.1016/s0006-291x(02)00336-4

Volt, H., Garcia, J. A., Doerrier, C., Diaz-Casado, M. E., Guerra-Librero, A., Lopez, L. C., et al. (2016). Same molecule but different expression: aging and sepsis trigger NLRP3 inflammasome activation, a target of melatonin. J. Pineal Res. 60, 193-205. doi: 10.1111/jpi.12303

Voordouw, B. C., Euser, R., Verdonk, R. E., Alberda, B. T., de Jong, F. H., Drogendijk, A. C., et al. (1992). Melatonin and melatonin-progestin combinations alter pituitary-ovarian function in women and can inhibit ovulation. J. Clin. Endocrinol. Metab. 74, 108-117. doi: 10.1210/jc.74. 1.108

Wang, X., Liu, Y., Sun, Y., Liu, W., and Jin, X. (2016). Blood brain barrier breakdown was found in non-infarcted area after 2-h MCAO. J. Neurol. Sci. 363, 63-68. doi: 10.1016/j.jns.2016.02.035

Wang, H., Li, L., Zhao, M., Chen, Y. H., Zhang, Z. H., Zhang, C., et al. (2011). Melatonin alleviates lipopolysaccharide-induced placental cellular stress response in mice. J. Pineal Res. 50, 418-426. doi: 10.1111/j.1600-079x. 2011.00860.x
Wang, X., Xue, G. X., Liu, W. C., Shu, H., Wang, M., Sun, Y., et al. (2017). Melatonin alleviates lipopolysaccharide-compromised integrity of blood-brain barrier through activating AMP-activated protein kinase in old mice. Aging Cell 16, 414-421. doi: 10.1111/acel.12572

Wang, H., Xu, D. X., Lv, J. W., Ning, H., and Wei, W. (2007). Melatonin attenuates lipopolysaccharide (LPS)-induced apoptotic liver damage in Dgalactosamine-sensitized mice. Toxicology 237, 49-57. doi: 10.1016/j.tox.2007. 04.021

Weiss, N., Miller, F., Cazaubon, S., and Couraud, P. O. (2009). The blood-brain barrier in brain homeostasis and neurological diseases. Biochim. Biophys. Acta 1788, 842-857. doi: 10.1016/j.bbamem.2008.10.022

Wong, C. S., Jow, G. M., Kaizaki, A., Fan, L. W., and Tien, L. T. (2014). Melatonin ameliorates brain injury induced by systemic lipopolysaccharide in neonatal rats. Neuroscience 267, 147-156. doi: 10.1016/j.neuroscience.2014. 02.032

Zhang, H., Liu, D., Wang, X., Chen, X., Long, Y., Chai, W., et al. (2013). Melatonin improved rat cardiac mitochondria and survival rate in septic heart injury. J. Pineal Res. 55, 1-6. doi: 10.1111/jpi.12033

Zhao, L., An, R., Yang, Y., Yang, X., Liu, H., Yue, L., et al. (2015). Melatonin alleviates brain injury in mice subjected to cecal ligation and puncture via attenuating inflammation, apoptosis, and oxidative stress: the role of SIRT1 signaling. J. Pineal Res. 59, 230-239. doi: 10.1111/jpi.12254

Zhao, Z., Hu, J., Gao, X., Liang, H., and Liu, Z. (2014). Activation of AMPK attenuates lipopolysaccharide-impaired integrity and function of blood-brain barrier in human brain microvascular endothelial cells. Exp. Mol. Pathol. 97, 386-392. doi: 10.1016/j.yexmp.2014.09.006

Zhou, X., Cao, Y., Ao, G., Hu, L., Liu, H., Wu, J., et al. (2014). CaMKKbetadependent activation of AMP-activated protein kinase is critical to suppressive effects of hydrogen sulfide on neuroinflammation. Antioxid. Redox Signal. 21, 1741-1758. doi: 10.1089/ars.2013.5587

Zhou, T., Zhao, L., Zhan, R., He, Q., Tong, Y., Tian, X., et al. (2014). Blood-brain barrier dysfunction in mice induced by lipopolysaccharide is attenuated by dapsone. Biochem. Biophys. Res. Commun. 453, 419-424. doi: 10.1016/j.bbrc. 2014.09.093

Conflict of Interest Statement: The authors declare that the research was conducted in the absence of any commercial or financial relationships that could be construed as a potential conflict of interest.

Copyright $\odot 2017$ Liu, Wang, Zhang, Chen and Jin. This is an open-access article distributed under the terms of the Creative Commons Attribution License (CC BY). The use, distribution or reproduction in other forums is permitted, provided the original author(s) or licensor are credited and that the original publication in this journal is cited, in accordance with accepted academic practice. No use, distribution or reproduction is permitted which does not comply with these terms. 